\title{
NEW ASPECTS OF THE ROLE OF DEVELOPMENT AND STRUC- TURE OF SOLAR ACTIVE REGIONS IN THE ARRANGEMENTS OF THE CORONA BASED ON ITS GEOMAGNETIC DISPLAYS
}

\author{
B. BednÁŘová-Nováková and J. HalenKa* \\ (Geophysical Institute of the Czechoslovak Academy of Sciences, Prague, C.S.S.R.)
}

\begin{abstract}
A BSTRACT
The coronal plasma, which is the cause of geomagnetic storms, can impinge on the Earth only when some coronal formation is pointing towards the Earth. If no such formation is directed towards the Earth, a period of geomagnetic calm follows.

It was found that certain coronal formations, governed clearly by the appropriate local magnetic fields, correspond to the individual stages of development of active centres. Above the sunspot groups, there occur either conical rarifications or cylindrical, condensed fluxes dependent on a relatively nonvariable, or unstable magnetic field. The filaments which outlive the sunspot period, provided they are located in floccular fields, are appropriate to streamers of helmet shape. The final stage of the active region, characterized by the presence of filaments only (called 'free filaments' by the authors), does not change the arrangement of the corona appreciably, i.e. the normal shape, or so-called minimum type. On the basis of the mentioned relations, it is possible to determine the shape and direction of so far currently unobservable coronal structures from chromospheric situations.

The mentioned facts enable a unified interpretation of the geomagnetic activity during the whole of the solar cycle to be made. The differences given for some types of storms (sporadic and recurrent, sudden and gradual commencement), may be explained by the various arrangements, occurrences or absences of local magnetic fields. The paper presents examples of chromospheric situations which were used, in some cases, at the Geophysical Institute in Prague for forecasting geomagnetic activity.
\end{abstract}

\section{DISCUSSION}

Fokker: I should like to inquire whether the author's results apply to magnetic storms with sudden commencement. There is general agreement between solar scientists that sudden commencements can, for the greatest part, be related to certain individual flares, notably such flares as produce type-IV radio outbursts. I wonder therefore why it should be necessary to invoke filaments as being directly connected with sudden commencements of geomagnetic storms.

Halenka: Our results do apply to magnetic storms with sudden commencements, as is explicitly stated in the text (see 'Unstable Filaments' and Appendix). We forecast SSCs successfully on the basis of spectrohelioscopic observations by Bednářová-Nováková (Travaux géophys. 1964, NČSAV Praha 1965, 277).

In our opinion, the solar process - apparently changes in magnetic fields -, the consequence of which is the emission of the corpuscular stream responsible for a geomagnetic storm, may also be accompanied by a flare if the conditions for its formation are favourable. The flare in itself is not the necessary condition for the emission, which may be seen from the fact that a great number of even SC storms are not preceded by any flare (Bednářová-Nováková, B., Travaux géophys. 1966, Academia Praha 1967, 477). A comparison of the occurrence of radiobursts and disappearing filaments shows that the emission of particles producing geomagnetic storms need not be apparent in the form of radiobursts (Halenka, J., Travaux géophys. 1965, Academia Praha 1966, 395).

* Presented by J. Halenka.

Kiepenheuer (ed.), Structure and Development of Solar Active Regions, 389. OI.A.U. 